\title{
Reflecting and reporting uncertainty in valuations: An alternative approach
}

\author{
Dr T M Havard and Mark Shepherd \\ Research Institute for the Built and Human Environment (BUHU) \\ University of Salford \\ Bridgewater Building \\ Salford \\ Greater Manchester \\ UK \\ M7 1NU \\ e-mail t.havard@salford.ac.uk
}

\section{Keywords}

Valuation practice, uncertainty, reporting

\begin{abstract}
A substantial body of work now exists to illustrate that valuations are open to uncertainty and that pinpoint accuracy in valuations cannot be expected (Hutchison et al, 1996, Crosby, 2000, Crosby et al, 1998). This viewpoint has been accepted by the professional body in the UK. As early as March 1994 The Mallinson Report argued that all valuations are uncertain, and recommended that valuers report conditions of "abnormal uncertainty". The more recent "Carsberg Report", which examined valuation practice and client-valuer relationships, also considered the accuracy and uncertainty issues: -

"The RICS should enter discussions with Investment Property Databank with a view to agreeing a means by which their data could be used to produce ongoing annual reports on the correlations between valuations and achieved prices as observed by IPD, and consider with the wider academic community how the data can be additionally analysed to provide better information on the currency of valuations....
\end{abstract}

The Carsberg Report, 2002, Recommendations, p1

The body of work and the Carsberg recommendations have been incorporated into a reworking of the "Red Book", now named the 'RICS Appraisal and Valuation Standards' which will be mandatory in practice from $1^{\text {st }}$ May 2003. In addition to this the RICS are commissioning research into expressing uncertainty in valuation reports, i.e. communicating the uncertainty that exists in valuations to clients so that they no longer rely on them as being accurate point estimates.

This paper examines the some of the possible alternatives that have been proposed to date.

Alternatives from overseas practice or academics include quoting a most probable price and a range of uncertainty, though objections have been raised to this. Mallinson and French (2000) in examining the issue, took a simulation type approach and suggested six items of information that needed to be conveyed in the valuation based upon the statistical analysis of the valuations produced by the process.

An alternative to these approaches is discussed in this paper. The approach arises out of interview research of valuers exploring the reasons for valuation failure. The technique employs a conventional valuation producing a single point estimate of value but requires the valuer to assess the conditions under which the valuation was undertaken using a Standard Reliability Assessment Matrix. This matrix has associated descriptors, similar to those used in classifications from other disciplines such as the Beaufort Scale and the Richter Scale, which enable the user of the valuation to assess the likely reliability of the valuation produced.

The practical advantages of the system are discussed.

Contact author 